\title{
Performance of Swarnadhara breeder hens supplemented with reduced levels of different copper forms
}

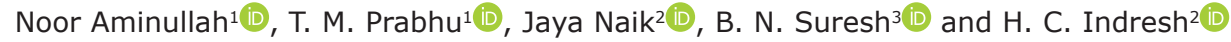 \\ 1. Department of Animal Nutrition, Veterinary College, Karnataka Veterinary, Animal and Fisheries Sciences University, \\ Bengaluru, Karnataka, India; 2. Department of Poultry Science, Veterinary College, Karnataka Veterinary, Animal and \\ Fisheries Sciences University, Bengaluru, Karnataka, India; 3. Department of Livestock Farm Complex, Veterinary \\ College, Karnataka Veterinary, Animal and Fisheries Sciences University, Hassan, Karnataka, India. \\ Corresponding author: Noor Aminullah, e-mail: aminullah.noor18@gmail.com \\ Co-authors: TMP: prabhutmann@gmail.com, JN: dr.jnaik2007@rediffmail.com, BNS: sureshann658@gmail.com, \\ HCI: drindreshpsc@gmail.com
}

Received: 15-01-2021, Accepted: 16-04-2021, Published online: 29-05-2021

doi: www.doi.org/10.14202/vetworld.2021.1371-1379 How to cite this article: Aminullah N, Prabhu TM, Naik J, Suresh BN, Indresh HC (2021) Performance of Swarnadhara breeder hens supplemented with reduced levels of different copper forms, Veterinary World, 14(5): 1371-1379.

\begin{abstract}
Background and Aim: Copper $(\mathrm{Cu})$ is a vital mineral involved in various physiological and biochemical processes, growth, and productivity of animals and birds. Birds can absorb only a small fraction of $\mathrm{Cu}$ and most is excreted, contaminating soil and aquatic environment which is toxic for microorganisms, plants, animals, and humans. This study evaluated the possibility of use of organic and nanoparticles sources of $\mathrm{Cu}$ to reduce supplementation level without compromising the performance of breeder hens.
\end{abstract}

Materials and Methods: A total of 224 Swarnadhara breeder hens were divided into seven treatment groups having four replicates in each. The basal diet (control) containing $20 \mathrm{ppm}$ inorganic $\mathrm{Cu}(100 \%$ of standard recommendation) and six test diets containing 20,15 , and $10 \mathrm{ppm}(100,75$, and $50 \%$ of standard recommendation) from $\mathrm{Cu}$ organic source, and 15,10 , and $5 \mathrm{ppm}(75,50$, and $25 \%)$ from $\mathrm{Cu}$ nanoparticles $(\mathrm{Cu}-\mathrm{NP})$, were prepared and offered to respective treatment groups for a duration of 20 weeks.

Results: The hen day egg production, hen housed egg production, feed conversion ratio egg mass, albumen index, yolk index, total fat content, and color score were not affected by the source and inclusion level of $\mathrm{Cu}$. The feed intake was significantly $(\mathrm{p}<0.05)$ lower at $15 \mathrm{ppm}$ and egg weight was significantly $(\mathrm{p}<0.05)$ higher at $10 \mathrm{ppm}$ Cu-NP supplemental level, but was non-significant in other treatment groups compared to control. The body weight gain was significantly $(\mathrm{p}<0.05)$ higher at $20 \mathrm{ppm}$ organic and $15 \mathrm{ppm} \mathrm{Cu}-\mathrm{NP}$ inclusion. The egg shape index and Haugh unit were significantly $(\mathrm{p}<0.05)$ lower at 10 and $15 \mathrm{ppm}$ of $\mathrm{Cu}-\mathrm{NP}$ inclusion level, respectively. The shell thickness was improved $(\mathrm{p}<0.05)$ at 20 and $15 \mathrm{ppm}$ organic and 15 and $10 \mathrm{ppm} \mathrm{Cu}-\mathrm{NP}$ inclusion level. The egg fertility rate was shown to be significantly $(\mathrm{p}<0.05)$ higher at $20 \mathrm{ppm}$ organic $\mathrm{Cu}$ inclusion group, but the hatchability based on total number of eggs set improved $(\mathrm{p}<0.05)$ at 20 and $15 \mathrm{ppm}$ organic $\mathrm{Cu}$ inclusion level while all treatment groups were comparable to control. The hatchability of fertilized egg and chick's quality significantly $(\mathrm{p}<0.05)$ improved, while embryonic and chick mortality after hatching before-sorting was significantly $(\mathrm{p}<0.05)$ reduced at $15 \mathrm{ppm}$ of $\mathrm{Cu}-\mathrm{NP}$ inclusion group.

Conclusion: It was concluded that the inorganic $\mathrm{Cu}$ can be replaced with $50 \%$ of organic or $25 \%$ of nanoparticles form of $\mathrm{Cu}$ without jeopardizing the breeder hens' productivity, egg quality characteristics, hatchability, and progeny.

Keywords: egg production, hatchability, nanoparticles, organic copper, poultry.

\section{Introduction}

The beneficial effect of copper $(\mathrm{Cu})$ on the development and functioning of bird is well documented [1-3] and recognized to serve as a cofactor for many enzymes such as cytochrome oxidase, lysyl oxidase [4], ceruloplasmin, and superoxide dismutase [5]. $\mathrm{Cu}$ is essential for growth, production, reproduction, and health of bird. However, the major ingredients included in poultry feed unable to supply the required

Copyright: Aminullah, et al. Open Access. This article is distributed under the terms of the Creative Commons Attribution 4.0 International License (http://creativecommons.org/licenses/ by/4.0/), which permits unrestricted use, distribution, and reproduction in any medium, provided you give appropriate credit to the original author(s) and the source, provide a link to the Creative Commons license, and indicate if changes were made. The Creative Commons Public Domain Dedication waiver (http:// creativecommons.org/publicdomain/zero/1.0/) applies to the data made available in this article, unless otherwise stated. quantity of $\mathrm{Cu}$ and its deficiency is common in practical feeding [6]. Therefore, dietary supplementation of $\mathrm{Cu}$ is essential $[7,8]$ to maintain the productivity of poultry. Further, the mineral's absorption and utilization is reported to be poor [8,9] and a large amount of trace elements is excreted in the manure of livestock and poultry [10] and pollute the environment. Poultry manure applied on nitrogen basis in agricultural land contains $500 \%$ more $\mathrm{Cu}$ content than the plants requirement for optimum growth $[8,11]$. The excess $\mathrm{Cu}$ can lead to high environmental concentration which is toxic for many plants and grazing animals [12], affect the balance of other minerals [13] and encourage unfavorable microorganisms in soil and water sources [14]. Therefore, biotechnological interventions are made to increase the $\mathrm{Cu}$ bioavailability and absorption and reduce its environmental 
emission to avoid causing harmful effects on animal, poultry, and human health.

It is acknowledged that the organic form of $\mathrm{Cu}$ can improve its utilization and minimize its level in excreta and environmental pollution [15] due to its better absorption and metabolism properties [16]. Nollet et al. [15] reported no difference in performance between broilers fed high levels of inorganic $\mathrm{Cu}$ and low level of organic chelated $\mathrm{Cu}$ but significantly $(p<0.05)$ lower fecal mineral excretion rates were observed in birds fed the organic form of $\mathrm{Cu}$. Gheisari et al. [17] concluded that organic minerals $(\mathrm{Cu}, \mathrm{Mn}$, and $\mathrm{Zn}$ ) at the dosage of $25-50 \%$ of inorganic minerals recommended level are sufficient to maintain performance and egg quality characteristics in laying hens.

Nanoparticle of $\mathrm{Cu}$ is another alternate form of $\mathrm{Cu}$ due to its novel properties such as their large surface-to-volume ratio [18], reactivity, and bioavailability [19] and prevents antagonism with other minerals [16]. Many studies have compared the availability of $\mathrm{Cu}$ nanoparticles ( $\mathrm{Cu}-\mathrm{NP})$ to $\mathrm{CuSO}_{4}$ normal size when supplemented in animal diet, suggesting that the $\mathrm{Cu}-\mathrm{NP}$ is better than its conventional inorganic form in enhancing the growth and performance of animals [20,21]. Kozłowski et al. [22] evaluated $\mathrm{Cu}-\mathrm{NP}$ at three different levels $(20,10$, and $2 \mathrm{mg} / \mathrm{kg}$ diet) to reduce its dietary supplemental level and concluded that $\mathrm{Cu}-\mathrm{NP}$ can be reduced by $50 \%$ without compromising the growth rate and antioxidant status of turkey birds. In the previous studies, it was shown that $\mathrm{Cu}$ in organic and nanoparticles forms can enhance its bioavailability, growth performance, egg production, hatchability, immune-modulation, reduce pathogen load, and improve health of breeder hens [13].

Very limited work has been done yet to study the dietary lower level of $\mathrm{Cu}$ using organic and nanoparticles form on layer egg production, egg quality characteristics, hatchability, and progeny. The aim of this study was to find the most biologically effective form of $\mathrm{Cu}$ with higher efficiency and reduce supplementation level without compromising the performance of breeder hens.

\section{Materials and Methods}

\section{Ethical approval}

All the procedures with regard to the management and care of the birds followed during the trial were approved (letter no.: VCH/IAEC/2020/01) by the Institutional Animal Ethics Committee of the University (KVAFSU, Bidar, Karnataka).

\section{Study period and location}

This experiment was conducted from July 2019 to November 2019 for a total of 20 weeks of production period at the Department of Poultry Science, Veterinary College, Hebbal, Bangalore, Karnataka, India, located at $\mathrm{N} 13.03^{\circ} \mathrm{N}$ and $77.60^{\circ} \mathrm{E}$.

\section{Experimental birds and management}

A genotype akin to country fowl named Swarnadhara breed was developed and released by
Veterinary College, KVAFSU, Bengaluru, for rural scavenging conditions in the year 2007. A total of 224 Swarnadhara breeder hens of 28 weeks age at the Department of Poultry Science, Veterinary College, Bengaluru, were utilized for the study. All the birds were wing banded and randomly assigned to seven treatment groups with four replicates having eight hens and one cock in each (32 hens per treatment group). The birds were reared in a deep litter system with standard managemental practices till 48 weeks of age. All the breeders were exposed to $15 \mathrm{~h}$ light. The experiment was conducted from $29^{\text {th }}$ to $48^{\text {th }}$ week of the birds' age, which constituted a total of 140 days and that was conveniently divided into five phases of 28 days each. All treatment groups received respective iso-nitrogenous and iso-caloric diets in all phases according to their production [23]. The daily required amount of feed was weighed and offered replicate-wise and recorded. Water was provided ad libitum during the trial period.

\section{Dietary treatments}

The basal diet was formulated as per the recommendations of Indian Council of Agricultural Research (ICAR) [13] nutrient requirements except $\mathrm{Cu}$ for poultry. The $\mathrm{Cu}-\mathrm{NP}$ were procured form $\mathrm{M} / \mathrm{s}$ Matrix Nano Pvt Ltd., New Delhi, and inorganic $\mathrm{Cu}$ sulfate and organic $\mathrm{Cu}$ as $\mathrm{Cu}$ proteinate from M/s Zeus-Biotech Pvt. Ltd., Mysuru, Karnataka, India. The $\mathrm{Cu}-\mathrm{NP}$ were prepared using induction coupled plasma (ICP) method having $98 \%$ purity and the particle size measured was $50-80 \mathrm{~nm}$. The basal diet was supplemented with $20 \mathrm{ppm} \mathrm{Cu}$ from inorganic $\mathrm{CuSO}_{4}$ $\left(\mathrm{T}_{1}\right)$ as this is the mainly used source of $\mathrm{Cu}$ in poultry feeding [9]. Treatment groups $\mathrm{T}_{2}, \mathrm{~T}_{3}$, and $\mathrm{T}_{4}$ were supplemented with 20,15 , and $10 \mathrm{ppm}$ levels (100, 75 , and $50 \%$ of standard recommended level) of $\mathrm{Cu}$ from organic source as $\mathrm{Cu}$ proteinate, respectively. Treatment groups $\mathrm{T}_{5}, \mathrm{~T}_{6}$, and $\mathrm{T}_{7}$ were supplemented at 15,10 , and 5 ppm levels $(75,50$, and $25 \%$ of standard recommended level) of Cu-NP, respectively (Table-1). The ingredient composition of experimental basal diet is presented in Table-2 and its chemical composition in Table-3. The $\mathrm{Cu}$ content in the feed samples was estimated using Inductively Coupled Plasma - Optical Emission Spectrophotometer; Perkin Elmer Optima 8000. The $\mathrm{Cu}$ content of basal and experimental diets is presented in Table-3. The respective feed was weighed and offered daily to all experimental groups. Each bird received $150 \mathrm{~g}$ feed daily in a single dose during the experimental period from $29^{\text {th }}$ to $48^{\text {th }}$ week of age.

\section{Production performance}

The daily egg production was recorded replicate wise and hen day egg production (HDEP) and hen housed egg production (HHEP) were calculated on percent basis. The feed conversion ratio (FCR) was calculated on the basis of feed consumed per unit egg mass produced. Egg mass was calculated period 
Table-1: Description of experimental groups.

\begin{tabular}{|c|c|c|c|}
\hline \multirow{2}{*}{$\begin{array}{l}\text { Experimental } \\
\text { group }\end{array}$} & \multirow{2}{*}{$\begin{array}{l}\text { IExperimental } \\
\text { diet }\end{array}$} & \multicolumn{2}{|c|}{ Cu supplementation level } \\
\hline & & $\begin{array}{l}\text { Total } \\
\text { Cu } \\
\text { (ppm) }\end{array}$ & $\begin{array}{c}\text { Compared } \\
\text { to standard } \\
\text { recommendation } \\
(\%)\end{array}$ \\
\hline $\mathrm{T}_{1}$ & $\begin{array}{l}\text { Basal diet+ } \\
\text { Inorganic Cu }\end{array}$ & 20 & 100 (Control) \\
\hline $\mathrm{T}_{2}$ & $\begin{array}{l}\text { Basal diet+ } \\
\text { Organic } \mathrm{Cu}^{1}\end{array}$ & 20 & 100 \\
\hline $\mathrm{T}_{3}$ & $\begin{array}{l}\text { Basal diet+ } \\
\text { Organic Cu }\end{array}$ & 15 & 75 \\
\hline $\mathrm{T}_{4}$ & $\begin{array}{l}\text { Basal diet+ } \\
\text { Organic Cu }\end{array}$ & 10 & 50 \\
\hline $\mathrm{T}_{5}$ & $\begin{array}{l}\text { Basal diet+ } \\
\text { Cu-NP2 }\end{array}$ & 15 & 75 \\
\hline $\mathrm{T}_{6}$ & $\begin{array}{l}\text { Basal diet+ } \\
\text { Cu-NP }\end{array}$ & 10 & 50 \\
\hline $\mathrm{T}_{7}$ & $\begin{array}{l}\text { Basal diet+ } \\
\text { Cu-NP }\end{array}$ & 5 & 25 \\
\hline
\end{tabular}

Table-2: Ingredients composition of basal diet (quantity/100 kg).

\begin{tabular}{lc}
\hline Ingredients & Part \\
\hline Yellow maize $(\mathrm{kg})$ & 58.86 \\
Soybean meal $(\mathrm{kg})$ & 22.5 \\
Deoiled rice bran $(\mathrm{kg})$ & 9.0 \\
Oyster shell grit $(\mathrm{kg})$ & 4.5 \\
Dicalcium phosphate $(\mathrm{kg})$ & 2.5 \\
Mineral mixture-without copper* $(\mathrm{kg})$ & 2.12 \\
Bacitracin methylene disalicylate $(\mathrm{g})$ & 70.0 \\
Vitamin premix** $(\mathrm{g})$ & 15.0 \\
Vitamin B complex*** $(\mathrm{g})$ & 25.0 \\
DL-Methionine $(\mathrm{g})$ & 100.0 \\
Hepatocare $(\mathrm{g})$ & 100.0 \\
Common salt $(\mathrm{g})$ & 210.0 \\
Total $(\mathrm{kg})$ & 100.0 \\
\hline
\end{tabular}

*Contains: Ca 32\%, P 9\%, Fe 2000 ppm, Iodin 0.01\%, $\mathrm{Mn} 0.4 \%$ and $\mathrm{Zn} 0.4 \%$. **Each gram contains: Vitamin A 82500 IU, Vitamin B2 50 mg, D3 12000 IU \& K 10 mg. $* * *$ Each gram contains: Vitamin B1 $4 \mathrm{mg}$, Vitamin B6 8 mg, Vitamin B12 40 mg, Vitamin E $40 \mathrm{mg}$, Calcium D pantothenate $40 \mathrm{mg}$, Niacin $60 \mathrm{mg}$

wise considering number of eggs produced and egg weight. The bird initial and period wise body weight was recorded to evaluate the body weight gain during the experiment.

\section{Egg quality characteristics}

Four eggs from each replicate were randomly selected on last day of each 28-day phase and evaluated for the external and internal quality characteristics. After the external characteristics study, each egg was broken and the entire contents were carefully emptied on a slab for metric measurement such as albumen index, yolk index, and Haugh unit score [24]. The egg yolk color was scored using matching technique Roche yolk color fan [25] and yolk fat content was evaluated as per AOAC [26] for the last phase of the experiment and expressed on dry matter basis.
Table-3: Chemical composition of the diet.

\begin{tabular}{|c|c|c|c|}
\hline \multirow{2}{*}{$\begin{array}{l}\text { Nutrient } \\
\mathrm{ME}(\mathrm{MJ} / \mathrm{kg})^{\mathrm{b}}\end{array}$} & \multicolumn{3}{|c|}{$\begin{array}{r}\text { Composition Total Cu content of } \\
\text { experimental diets }\end{array}$} \\
\hline & 11.67 & Diet & $\mathrm{mg} / \mathrm{kg}$ \\
\hline Crude Protein $(\%)^{\mathrm{a}}$ & 16.65 & Basal diet* & 8.03 \\
\hline Calcium $(\%)^{a}$ & 3.03 & $\mathrm{~T}_{1}$ & 28.04 \\
\hline Total phosphorus (\%) & 0.77 & $\mathrm{~T}_{2}^{1}$ & 28.07 \\
\hline Lysine $(\%)^{\mathrm{b}}$ & 0.78 & $\mathrm{~T}_{3}^{2}$ & 23.03 \\
\hline Methionine $(\%)^{\mathrm{b}}$ & 0.41 & $\mathrm{~T}_{4}^{3}$ & 18.05 \\
\hline Selenium $(\mathrm{ppm})^{\mathrm{b}}$ & 0.25 & $\mathrm{~T}_{5}^{4}$ & 23.06 \\
\hline Zinc $(p p m)^{a}$ & 86.51 & $\mathrm{~T}_{6}^{\mathrm{s}}$ & 18.05 \\
\hline $\mathrm{Fe}(\mathrm{ppm})^{\mathrm{a}}$ & 103.65 & $\mathrm{~T}_{7}^{\circ}$ & 13.04 \\
\hline $\mathrm{Mn}(\mathrm{ppm})^{\mathrm{a}}$ & 59.55 & & \\
\hline Vitamin A $(\mathrm{IU} / \mathrm{kg})^{\mathrm{b}}$ & 14637.0 & & \\
\hline Vitamin D3 (IU/kg) & 1846.00 & & \\
\hline Vitamin E $(\mathrm{IU} / \mathrm{kg})^{\mathrm{b}}$ & 91.37 & & \\
\hline Thiamin $(\mathrm{mg} / \mathrm{kg})^{\mathrm{b}}$ & 4.87 & & \\
\hline Riboflavin $(\mathrm{mg} / \mathrm{kg})^{\mathrm{b}}$ & 12.95 & & \\
\hline Pyridoxine $(\mathrm{mg} / \mathrm{kg})^{\mathrm{b}}$ & 6.25 & & \\
\hline $\begin{array}{l}\text { Cyanocoblamine } \\
(\mathrm{mg} / \mathrm{kg})^{\mathrm{b}}\end{array}$ & 0.45 & & \\
\hline
\end{tabular}

Copper $=\mathrm{Cu}$, aAnalyzed value, ${ }^{\mathrm{b} C a l c u l a t e d}$ value, $*$ Without addition of supplemental $\mathrm{Cu}$

\section{Fertility, hatchability, and progeny study}

Egg produced during last 3 consecutive days of each phase were pooled and set replicate-wise for fertility, hatchability, and progeny study in incubator. The egg candled on day $18^{\text {th }}$ of incubation to sort and record fertile and infertile egg. Number of embryonic mortality, chick mortality after hatching-before sorting and live-good quality chicks were recorded on $21^{\text {st }}$ day of incubation. For progeny evaluation, four chicks per replicate (16 per treatment group) were randomly selected and subjected for quality evaluation using the method described by Tona et al. [27] with scoring based on activity, feathering and appearance, condition of eyes, conformation of legs, condition of navel area, remaining yolk sac, and status of the yolk membranes.

\section{Statistical analysis}

The data were subjected to analysis of variance analysis using Statistical Package for the Social Sciences version16 (SPSS Inc., USA). The findings were subjected to test the $\mathrm{H}_{\mathrm{o}}$ (Null hypothesis) at designed level of significance ( $\mathrm{p} \leq 0.05)$. If the $\mathrm{p} \leq 0.05$, value of traits was considered as a significant difference among the treatment groups. Mean comparison was made using Tukey post hoc test.

\section{Results}

The egg production in terms of HDEP and HHEP, feed intake, FCR, body weight gain, egg weight, and egg mass is summarized in Table- 4 . The pooled data over the periods showed that different sources of $\mathrm{Cu}$ at different dietary inclusion level have no significant ( $p>0.05$ ) effect on egg production, FCR, and egg mass of the hens. The feed intake was significantly $(p<0.05)$ lower in $T_{5}$ and egg weight was significantly $(\mathrm{p}<0.05)$ higher in $\mathrm{T}_{6}$ as compared to control $\left(\mathrm{T}_{1}\right)$, 
Table-4: Egg production, feed intake, feed conversion ratio, egg weight, egg mass and body weight gain influenced by different treatments.

\begin{tabular}{|c|c|c|c|c|c|c|c|c|c|}
\hline $\begin{array}{l}\text { Treatment } \\
\text { group }\end{array}$ & $\begin{array}{l}\text { Cu level } \\
(\%) \text { of } \\
\text { requirement }\end{array}$ & Cu source & $\begin{array}{c}\text { Hen } \\
\text { day egg } \\
\text { production } \\
(\%)\end{array}$ & $\begin{array}{c}\text { Hen } \\
\text { housed } \\
\text { egg } \\
\text { production } \\
(\%)\end{array}$ & $\begin{array}{c}\text { Feed intake } \\
\text { (g/day/ } \\
\text { hen) }\end{array}$ & $\begin{array}{c}\text { FCR (kg } \\
\text { feed } \\
\text { intake/kg } \\
\text { egg mass) }\end{array}$ & $\begin{array}{c}\text { Egg } \\
\text { weight } \\
\text { (g) }\end{array}$ & $\begin{array}{c}\text { Egg } \\
\text { mass } \\
\text { (g/ } \\
\text { day/ } \\
\text { hen) }\end{array}$ & $\begin{array}{c}\text { Body } \\
\text { weight } \\
\text { gain (g) }\end{array}$ \\
\hline $\mathrm{T}_{1}$ & 100 & Inorganic & $\begin{array}{c}64.06 \pm \\
1.66\end{array}$ & $\begin{array}{c}63.06 \pm \\
1.66\end{array}$ & $\begin{array}{c}149.50 \pm \\
0.10^{\mathrm{b}}\end{array}$ & $\begin{array}{c}3.80 \pm \\
0.07\end{array}$ & $\begin{array}{c}58.26 \pm \\
0.66^{\mathrm{b}}\end{array}$ & $\begin{array}{c}39.27 \pm \\
0.59\end{array}$ & $\begin{array}{c}50.13 \pm \\
10.60^{\mathrm{b}}\end{array}$ \\
\hline $\mathrm{T}_{2}$ & 100 & Organic & $\begin{array}{c}64.55 \pm \\
1.33\end{array}$ & $\begin{array}{c}64.55 \pm \\
1.33\end{array}$ & $\begin{array}{c}149.78 \pm \\
0.05^{\mathrm{b}}\end{array}$ & $\begin{array}{c}3.81 \pm \\
0.06\end{array}$ & $\begin{array}{c}59.04 \pm \\
0.60^{\mathrm{ab}}\end{array}$ & $\begin{array}{c}39.52 \pm \\
0.65\end{array}$ & $\begin{array}{c}99.63 \pm \\
10.93^{a}\end{array}$ \\
\hline $\mathrm{T}_{3}$ & 75 & Organic & $\begin{array}{c}65.57 \pm \\
1.55\end{array}$ & $\begin{array}{c}63.82 \pm \\
1.81\end{array}$ & $\begin{array}{c}149.58 \pm \\
0.13^{\mathrm{b}}\end{array}$ & $\begin{array}{c}3.72 \pm \\
0.05\end{array}$ & $\begin{array}{c}59.99 \pm \\
0.66^{\mathrm{ab}}\end{array}$ & $\begin{array}{c}39.13 \pm \\
0.84\end{array}$ & $\begin{array}{c}70.41 \pm \\
9.69^{\mathrm{b}}\end{array}$ \\
\hline $\mathrm{T}_{4}$ & 50 & Organic & $\begin{array}{c}62.38 \pm \\
1.60\end{array}$ & $\begin{array}{c}62.39 \pm \\
1.60\end{array}$ & $\begin{array}{c}149.29 \pm \\
0.06^{\mathrm{b}}\end{array}$ & $\begin{array}{c}3.83 \pm \\
0.06\end{array}$ & $\begin{array}{c}60.03 \pm \\
0.51^{\mathrm{ab}}\end{array}$ & $\begin{array}{c}39.19 \pm \\
0.74\end{array}$ & $\begin{array}{c}77.56 \pm \\
12.23^{b}\end{array}$ \\
\hline $\mathrm{T}_{5}$ & 75 & Nanoparticles & $\begin{array}{c}64.19 \pm \\
1.45\end{array}$ & $\begin{array}{c}63.97 \pm \\
1.42\end{array}$ & $\begin{array}{c}145.34 \pm \\
0.56^{\mathrm{a}}\end{array}$ & $\begin{array}{c}3.78 \pm \\
0.09\end{array}$ & $\begin{array}{c}60.03 \pm \\
0.51^{\mathrm{ab}}\end{array}$ & $\begin{array}{c}39.80 \pm \\
0.88\end{array}$ & $\begin{array}{c}96.31 \pm \\
10.65^{\mathrm{a}}\end{array}$ \\
\hline $\mathrm{T}_{6}$ & 50 & Nanoparticles & $\begin{array}{c}63.13 \pm \\
1.52\end{array}$ & $\begin{array}{c}63.13 \pm \\
1.52\end{array}$ & $\begin{array}{c}149.63^{ \pm} \\
0.07^{b}\end{array}$ & $\begin{array}{c}3.83 \pm \\
0.07\end{array}$ & $\begin{array}{c}60.84 \pm \\
0.67^{a}\end{array}$ & $\begin{array}{c}39.38 \pm \\
0.75\end{array}$ & $\begin{array}{c}91.20 \pm \\
10.14^{\mathrm{b}}\end{array}$ \\
\hline $\mathrm{T}_{7}$ & 25 & Nanoparticles & $\begin{array}{c}64.38 \pm \\
1.18\end{array}$ & $\begin{array}{c}64.38 \pm \\
1.18\end{array}$ & $\begin{array}{c}149.59 \pm \\
0.08\end{array}$ & $\begin{array}{c}3.78 \pm \\
0.06\end{array}$ & $\begin{array}{c}59.65^{ \pm} \\
0.61^{\mathrm{ab}}\end{array}$ & $\begin{array}{c}39.74 \pm \\
0.65\end{array}$ & $\begin{array}{c}78.44 \pm \\
9.12^{\mathrm{b}}\end{array}$ \\
\hline SEM & & & 3.546 & 3.631 & 0.462 & 0.168 & 2.881 & 1.760 & 71.375 \\
\hline p-value & & & 0.824 & 0.959 & $<0.001$ & 0.939 & 0.081 & 0.986 & 0.015 \\
\hline
\end{tabular}

Mean values bearing different superscripts within the column differ significantly $(p<0.05), C u=C o p p e r, F C R=F e e d$ conversion ratio

while comparable with all other treatment groups. The weight gain was significantly $(\mathrm{p}<0.05)$ higher in $\mathrm{T}_{2}$ and $\mathrm{T}_{5}$ as compared to control but all other treatment groups were non-significant $(\mathrm{p}>0.05)$ with each other.

Egg external and internal quality characteristics, including shape index, albumen index, yolk index, yolk fat content, yolk color score, and shell thickness are presented in Table-5. The pooled data over the experimental periods indicated non-significant $(p>0.05)$ variation in albumen index, yolk index, yolk fat content, and yolk color score in different treatment groups. The shape index was significantly $(\mathrm{p}<0.05)$ lower in $\mathrm{T}_{6}$ as compared to control, $\mathrm{T}_{2}$ and $\mathrm{T}_{3}$, while Haugh unit was significantly $(\mathrm{p}<0.05)$ lower in $\mathrm{T}_{5}$ compared to control and comparable with other treatment groups. The eggshell thickness was lesser in control, significantly $(\mathrm{p}<0.05)$ improved in $\mathrm{T}_{2}, \mathrm{~T}_{3}$, and $\mathrm{T}_{6}$ as compared to control but highest $(\mathrm{p}<0.05)$ in $\mathrm{T}_{5}$ as compared to all treatment groups.

The egg fertility and hatchability based on total and fertilized egg set, percentage of embryonic mortality and chick mortality after hatching-before sorting and chick's quality are summarized in Table-6. The egg fertility was significantly $(\mathrm{p}<0.05)$ higher in $\mathrm{T}_{2}$ as compared to all other treatment groups except of $\mathrm{T}_{3}$. No significant effect of $\mathrm{Cu}-\mathrm{NP}$ observed on egg fertility rate. The hatchability rate based on total number of eggs set was similar to that trend of fertility and no significant $(p>0.05)$ difference was observed in treatment groups as compared to control. Hatchability percentage based on fertilized egg set was significantly $(\mathrm{p}<0.05)$ higher in $\mathrm{T}_{3}, \mathrm{~T}_{5}$, and $\mathrm{T}_{6}$ as compared to control, but comparable with other treatment groups. Similarly, the chick's and embryonic mortality rate was also significantly $(\mathrm{p}<0.05)$ lower in $\mathrm{T}_{5}$ and $\mathrm{T}_{6}$ as compared to $\mathrm{T}_{4}$ and $\mathrm{T}_{7}$. The chicks in $\mathrm{T}_{5}$ treatment group had significantly $(\mathrm{p}<0.05)$ higher Tona score as compared to control $\left(\mathrm{T}_{1}\right), \mathrm{T}_{2}, \mathrm{~T}_{3}$, and $\mathrm{T}_{4}$, but comparable with $\mathrm{T}_{6}$ and $\mathrm{T}_{7}$.

\section{Discussion}

\section{Production performance}

In spite of reduced supplemental level of organic and nano-forms of $\mathrm{Cu}$ as compared to standard recommended level of normal form of inorganic $\mathrm{CuSO}_{4}$, the egg production, FCR, and egg mass are maintained without any adverse effect among different treatment groups as compared to control. Our study confirmed the better efficiency of organic [28] and nanoparticles [29] forms of $\mathrm{Cu}$ in egg production. The current result is in close agreement with the findings of Gheisari et al. [17] who illustrated that inclusion of organic minerals mixture ( $\mathrm{Zn}, \mathrm{Mn}$, and $\mathrm{Cu}$ ) at the level $50-75 \%$ less than NRC [30] recommendations is optimum to obtain optimal egg production, FCR, and egg mass. Saldanha et al. [31] reported that the organic minerals mixture $(\mathrm{Zn}, \mathrm{Mn}$, and $\mathrm{Cu})$ or organic $\mathrm{Cu}$ [32] dietary supplementation level can be reduced by $30 \%$ without compromising the production performance of laying hens besides reducing $\mathrm{Cu}$ excretion to the environment. Our result also confirm with Ramesh [33] that $\mathrm{Cu}-\mathrm{NP}$ dietary supplementation can be reduced by $75 \%$ as compared to its inorganic bulk form without compromising the egg production and egg mass. However, the findings are in contrast with Londero et al. [34] who concluded increased egg production due to addition of organic minerals in laying hens' diets.

Significant $(p<0.05)$ variation observed in respect of egg weight, feed intake, and body weight gain due to the source and inclusion level of $\mathrm{Cu}$ in different treatment groups. The egg weight was only 
Available at www.veterinaryworld.org/Vol.14/May-2021/40.pdf

Table-5: Internal and external egg quality characteristics influenced by different treatments.

\begin{tabular}{|c|c|c|c|c|c|c|c|c|c|}
\hline $\begin{array}{l}\text { Treatment } \\
\text { group }\end{array}$ & $\begin{array}{c}\mathrm{Cu} \\
\text { level }(\%) \\
\text { of } \\
\text { requirement }\end{array}$ & $\begin{array}{l}\text { Cu source } \\
\text { t }\end{array}$ & $\begin{array}{l}\text { Shape } \\
\text { index }\end{array}$ & $\begin{array}{l}\text { Albumen } \\
\text { index }\end{array}$ & Yolk index & $\begin{array}{c}\text { Haugh } \\
\text { unit } \\
\text { score }\end{array}$ & $\begin{array}{c}\text { Shell } \\
\text { thickness } \\
(\mathrm{mm})\end{array}$ & $\begin{array}{c}\text { Yolk fat } \\
\text { content }(\%) \\
\text { dry matter } \\
\text { basis }\end{array}$ & $\begin{array}{l}\text { Yolk } \\
\text { color } \\
\text { score }\end{array}$ \\
\hline$\overline{T_{1}}$ & 100 & Inorganic & $\begin{array}{c}74.46 \pm \\
0.52^{\mathrm{b}}\end{array}$ & $\begin{array}{c}0.10 \pm \\
0.00\end{array}$ & $\begin{array}{c}0.44 \pm \\
0.01\end{array}$ & $\begin{array}{c}86.42 \pm \\
0.56^{\mathrm{a}}\end{array}$ & $\begin{array}{l}0.32 \pm \\
0.00^{a}\end{array}$ & $\begin{array}{c}59.49 \pm \\
0.99\end{array}$ & $\begin{array}{c}7.28 \pm \\
0.11\end{array}$ \\
\hline $\mathrm{T}_{2}$ & 100 & Organic & $\begin{array}{c}75.27 \pm \\
0.42^{\mathrm{b}}\end{array}$ & $\begin{array}{c}0.10 \pm \\
0.00\end{array}$ & $\begin{array}{c}0.43 \pm \\
0.01\end{array}$ & $\begin{array}{c}84.02 \pm \\
0.54^{\mathrm{ab}}\end{array}$ & $\begin{array}{l}0.34 \pm \\
0.00^{\mathrm{b}}\end{array}$ & $\begin{array}{c}57.94 \pm \\
1.89\end{array}$ & $\begin{array}{c}7.33 \pm \\
0.10\end{array}$ \\
\hline $\mathrm{T}_{3}$ & 75 & Organic & $\begin{array}{c}74.46 \pm \\
0.41^{\mathrm{b}}\end{array}$ & $\begin{array}{c}0.09 \pm \\
0.00\end{array}$ & $\begin{array}{c}0.42 \pm \\
0.01\end{array}$ & $\begin{array}{c}84.41 \pm \\
0.54^{\mathrm{ab}}\end{array}$ & $\begin{array}{c}0.34 \pm \\
0.00^{\mathrm{b}}\end{array}$ & $\begin{array}{c}58.25 \pm \\
1.54\end{array}$ & $\begin{array}{c}7.31 \pm \\
0.09\end{array}$ \\
\hline $\mathrm{T}_{4}$ & 50 & Organic & $\begin{array}{c}73.65^{ \pm} \\
0.94^{\mathrm{ab}}\end{array}$ & $\begin{array}{c}0.10 \pm \\
0.00\end{array}$ & $\begin{array}{c}0.43 \pm \\
0.00\end{array}$ & $\begin{array}{c}85.12 \pm \\
0.56^{\mathrm{ab}}\end{array}$ & $\begin{array}{l}0.33 \pm \\
0.00^{\mathrm{ab}}\end{array}$ & $\begin{array}{c}59.01 \pm \\
0.60\end{array}$ & $\begin{array}{c}7.17 \pm \\
0.10\end{array}$ \\
\hline $\mathrm{T}_{5}$ & 75 & Nanoparticles & $\begin{array}{c}73.19 \pm \\
0.34^{\mathrm{ab}}\end{array}$ & $\begin{array}{c}0.09 \pm \\
0.00\end{array}$ & $\begin{array}{c}0.43 \pm \\
0.00\end{array}$ & $\begin{array}{c}82.79 \pm \\
0.58^{\mathrm{b}}\end{array}$ & $\begin{array}{l}0.36 \pm \\
0.00^{c}\end{array}$ & $\begin{array}{c}59.05 \pm \\
0.89\end{array}$ & $\begin{array}{c}7.16 \pm \\
0.09\end{array}$ \\
\hline $\mathrm{T}_{6}$ & 50 & Nanoparticles & $\begin{array}{c}70.35 \pm \\
1.45^{\mathrm{a}}\end{array}$ & $\begin{array}{c}0.10 \pm \\
0.0\end{array}$ & $\begin{array}{c}0.44 \pm \\
0.01\end{array}$ & $\begin{array}{c}85.96 \pm \\
1.00^{\mathrm{a}}\end{array}$ & $\begin{array}{l}0.34 \pm \\
0.00^{b}\end{array}$ & $\begin{array}{c}59.78 \pm \\
3.30\end{array}$ & $\begin{array}{c}7.34 \pm \\
0.98\end{array}$ \\
\hline $\mathrm{T}_{7}$ & 25 & Nanoparticles & $74.06 \pm 0.92^{\mathrm{b}}$ & $\begin{array}{c}0.09 \pm \\
0.00\end{array}$ & $\begin{array}{c}0.43 \pm \\
0.01\end{array}$ & $\begin{array}{c}85.20 \pm \\
0.65^{\mathrm{ab}}\end{array}$ & $\begin{array}{l}0.34 \pm \\
0.00^{\mathrm{ab}}\end{array}$ & $\begin{array}{c}59.57 \pm \\
2.98\end{array}$ & $\begin{array}{c}7.31 \pm \\
0.93\end{array}$ \\
\hline $\begin{array}{l}\text { SEM } \\
\text { p-value }\end{array}$ & & & $\begin{array}{l}3.869 \\
<0.001\end{array}$ & $\begin{array}{l}0.017 \\
0.068\end{array}$ & $\begin{array}{l}0.024 \\
0.842\end{array}$ & $\begin{array}{l}3.128 \\
0.002\end{array}$ & $\begin{array}{c}0.017 \\
<0.001\end{array}$ & $\begin{array}{l}2.136 \\
0.993\end{array}$ & $\begin{array}{l}0.483 \\
0.792\end{array}$ \\
\hline
\end{tabular}

Mean values bearing different superscripts within the column differ significantly $(p<0.05), C u=C o p p e r, F C R=F e e d$ conversion ratio

Table-6: Fertility, hatchability, chick's quality, and breeder hen survivability as influenced by different treatment.

\begin{tabular}{|c|c|c|c|c|c|c|c|c|c|}
\hline $\begin{array}{l}\text { Treatment } \\
\text { group }\end{array}$ & $\begin{array}{c}\mathrm{Cu} \\
\text { level }(\%) \\
\text { of } \\
\text { requirement }\end{array}$ & Cu Source & $\begin{array}{c}\text { Fertility } \\
(\%)\end{array}$ & $\begin{array}{c}\text { Hatchability } \\
(\%) \text { based } \\
\text { on total egg } \\
\text { set }\end{array}$ & $\begin{array}{c}\text { Hatchability } \\
\text { (\%) based } \\
\text { on fertile } \\
\text { egg set }\end{array}$ & $\begin{array}{c}\text { Chick } \\
\text { mortality } \\
\text { (\%) after } \\
\text { hatching } \\
\text { before } \\
\text { sorting }\end{array}$ & $\begin{array}{c}\text { Embryonic } \\
\text { mortality } \\
(\%)\end{array}$ & $\begin{array}{c}\text { Chick } \\
\text { quality } \\
\text {-Tona } \\
\text { score } \\
(\%)\end{array}$ & $\begin{array}{c}\text { Breeder } \\
\text { hens' } \\
\text { survivability }\end{array}$ \\
\hline $\mathrm{T}_{1}$ & 100 & Inorganic & $\begin{array}{c}85.87 \pm \\
0.83^{\text {acd }}\end{array}$ & $\begin{array}{c}74.90 \pm \\
1.07^{\mathrm{ab}}\end{array}$ & $\begin{array}{c}83.78 \pm \\
1.02^{\mathrm{a}}\end{array}$ & & $\begin{array}{l}7.05 \pm \\
0.95^{\mathrm{ab}}\end{array}$ & $\begin{array}{c}89.50 \pm \\
1.06^{\mathrm{ad}}\end{array}$ & $\begin{array}{c}98.75 \pm \\
0.86^{\mathrm{ab}}\end{array}$ \\
\hline $\mathrm{T}_{2}$ & 100 & Organic & $\begin{array}{c}91.89 \pm \\
0.84^{b}\end{array}$ & $\begin{array}{c}80.74 \pm \\
1.23^{\mathrm{a}}\end{array}$ & $\begin{array}{c}87.36 \pm \\
1.00^{\text {acd }}\end{array}$ & $\begin{array}{l}5.62 \pm \\
1.06^{\mathrm{ab}}\end{array}$ & $\begin{array}{l}7.34 \pm \\
0.90^{\mathrm{ab}}\end{array}$ & $\begin{array}{c}92.45 \pm \\
0.86^{\mathrm{cd}}\end{array}$ & $\begin{array}{c}100.00 \pm \\
0.00^{\mathrm{b}}\end{array}$ \\
\hline $\mathrm{T}_{3}$ & 75 & Organic & $\begin{array}{c}89.00 \pm \\
1.19^{\mathrm{bc}}\end{array}$ & $\begin{array}{c}80.16 \pm \\
1.22^{\mathrm{a}}\end{array}$ & $\begin{array}{c}89.00 \pm \\
1.30^{\text {cd }}\end{array}$ & $\begin{array}{l}4.47 \pm \\
1.12^{\mathrm{ab}}\end{array}$ & $\begin{array}{l}6.27 \pm \\
0.80^{\text {ab }}\end{array}$ & $\begin{array}{c}92.12 \pm \\
0.95^{\mathrm{ac}}\end{array}$ & $\begin{array}{c}97.18 \pm \\
1.15^{\mathrm{a}}\end{array}$ \\
\hline $\mathrm{T}_{4}$ & 50 & Organic & $\begin{array}{c}85.03 \pm \\
1.02^{\mathrm{ac}}\end{array}$ & $\begin{array}{c}72.64 \pm \\
1.44^{b}\end{array}$ & $\begin{array}{c}83.87 \pm \\
0.92^{\mathrm{a}}\end{array}$ & $\begin{array}{l}6.37 \pm \\
1.21^{\mathrm{ab}}\end{array}$ & $\begin{array}{c}7.93 \pm \\
0.90^{\mathrm{a}}\end{array}$ & $\begin{array}{c}88.60 \pm \\
1.17^{\mathrm{a}}\end{array}$ & $\begin{array}{c}100.00 \pm \\
0.00^{\mathrm{b}}\end{array}$ \\
\hline $\mathrm{T}_{5}$ & 75 & Nanoparticles & $\begin{array}{c}81.70 \pm \\
0.97^{a}\end{array}$ & $\begin{array}{c}74.73 \pm \\
1.51^{\mathrm{b}}\end{array}$ & $\begin{array}{c}94.23 \pm \\
1.29^{\mathrm{b}}\end{array}$ & $\begin{array}{c}2.65^{ \pm} \\
0.84^{b}\end{array}$ & $\begin{array}{c}3.57 \pm \\
0.91^{b}\end{array}$ & $\begin{array}{c}96.63 \pm \\
0.96^{\mathrm{b}}\end{array}$ & $\begin{array}{c}99.84 \pm \\
0.15^{\mathrm{b}}\end{array}$ \\
\hline $\mathrm{T}_{6}$ & 50 & Nanoparticles & $\begin{array}{c}83.25 \pm \\
1.48^{\text {ad }}\end{array}$ & $\begin{array}{c}75.56 \pm \\
1.72^{\mathrm{ab}}\end{array}$ & $\begin{array}{c}92.09 \pm \\
0.98^{\text {bd }}\end{array}$ & $\begin{array}{c}4.22 \pm \\
0.88^{b}\end{array}$ & $\begin{array}{c}3.57 \pm \\
0.91^{b}\end{array}$ & $\begin{array}{c}95.67 \pm \\
0.69^{\mathrm{bc}}\end{array}$ & $\begin{array}{c}100.00^{ \pm} \\
0.00^{\mathrm{b}}\end{array}$ \\
\hline $\mathrm{T}_{7}$ & 25 & Nanoparticles & $\begin{array}{c}86.59 \pm \\
1.30^{\mathrm{dc}}\end{array}$ & $\begin{array}{c}75.70 \pm \\
1.54^{\mathrm{ab}}\end{array}$ & $\begin{array}{c}85.85 \pm \\
1.23^{\text {ac }}\end{array}$ & $\begin{array}{l}5.37 \pm \\
0.12^{\mathrm{ab}}\end{array}$ & $\begin{array}{c}8.54 \pm \\
1.00^{\mathrm{a}}\end{array}$ & $\begin{array}{c}93.87 \pm \\
0.87^{\text {bc }}\end{array}$ & $\begin{array}{c}100.00 \pm \\
0.00^{\mathrm{b}}\end{array}$ \\
\hline SEM & & & 2.667 & 3.352 & 2.674 & 2.562 & 2.191 & 4.327 & 1.308 \\
\hline $\mathrm{p}$-value & & & $<0.001$ & $<0.001$ & $<0.001$ & 0.005 & $<0.001$ & $<0.001$ & $<0.001$ \\
\hline
\end{tabular}

Mean values bearing different superscripts within the column differ significantly $(p<0.05), \mathrm{Cu}=\mathrm{Copper}, \mathrm{FCR}=\mathrm{Feed}$ conversion ratio

significantly higher in $\mathrm{T}_{6}$ than in $\mathrm{T}_{1}$ group. The feed intake was significantly $(\mathrm{P}<0.05)$ lowest in $\mathrm{T}_{5}$ as compared to all other treatment groups while the FCR was comparable among all the groups. This reflects improved feed efficiency at $15 \mathrm{ppm}$ inclusion level of $\mathrm{Cu}-\mathrm{NP}$ in the diet as compared to 10 and $5 \mathrm{ppm}$ but all other treatment groups were comparable. Similarly, Ramesh [33] demonstrated that Cu-NP dietary inclusion at $25-50 \%$ of requirement can reduce feed intake without compromising the FCR in laying hens. The lower feed intake without affecting FCR can be attributed to improved feed efficiency and energy utilization [29]. $\mathrm{Cu}$ plays a key role in cytochrome $\mathrm{C}$ oxidase enzyme which is essential for the production of adenosine triphosphate within the cell resulting in efficient energy utilization [35]. It is documented that the $\mathrm{Cu}-\mathrm{NP}$ is crystalline in nature and has strong antimicrobial activity against both Gram-positive and Gram-negative bacteria [36], even showed the most efficient antimicrobial activity among the different forms of $\mathrm{Cu}[37,38]$. This may be the reason for better gut health and improved nutrients utilization in $\mathrm{T}_{5}$ treatment group. In contrast, the organic form of $\mathrm{Cu}$ had no effect on feed intake as compared to control. The current result is in consistent with Yenice et al. [39] and Attia et al. [40] who reported that organic $\mathrm{Cu}$ form has no significant effect on feed consumption in laying hens.

The pooled data over the periods showed a significant $(\mathrm{p}<0.05)$ increase in egg weight at $50 \%$ 
supplemental level of $\mathrm{Cu}-\mathrm{NP}\left(\mathrm{T}_{6}\right)$ as compared to control $\left(\mathrm{T}_{1}\right)$, while comparable with all other treatment groups. The findings are in agreement with Attia et al. [40] who observed no significant effect of organic source of $\mathrm{Cu}$ on egg weight, but is contradicted with Lim and Paik [41] who found that organic form of $\mathrm{Cu}$ can increase the egg weight in laying hens. In the previous study, Ramesh [33] reported no significant increase of egg weight due to Cu-NP dietary supplementation in Vanaraja breeder hens, while our result showed significantly $(\mathrm{p}<0.05)$ higher egg weight at $10 \mathrm{ppm}$ inclusion level. The $10 \mathrm{ppm}$ of $\mathrm{Cu}-\mathrm{NP}$ found to be the optimum level for egg weight. The better egg weight is attributed to higher fat content of egg yolk $(59.78 \%)$ which is slightly higher compared to other treatment groups. Pesti and Bakalli [42] also suggested that $\mathrm{Cu}$ supplementation can affect reproductive physiology and lipid metabolism in layer bird.

The pooled values of body weight gain over the periods were significantly $(p<0.05)$ different among treatment groups. The weight gain found to be dose-dependent response, but it could maintain the body weight at 50 and $25 \%$ inclusion level of organic and $\mathrm{Cu}-\mathrm{NP}$ as compared to control, respectively. In the previous studies, it was reported that the growth performance can be maintained with $75 \%$ reduction of $\mathrm{Cu}-\mathrm{NP}$ as compared with bulk form of $\mathrm{CuSO}_{4}$ supplemental level in growing chicks [43] and laying hens [33]. The result clearly demonstrates that dietary $\mathrm{CuSO}_{4}$ inorganic form can be replaced with $25 \%$ inclusion level of $\mathrm{Cu}-\mathrm{NP}$ to maintain the body weight gain in laying hens. In contrast, it was reported that organic source and dose of minerals have no significant ( $p>0.05$ ) effect on body weight changes in laying hens [39] and duck [44] as compared to inorganic form of $\mathrm{CuSO}_{4}$.

\section{Egg quality characteristics}

The present study indicated significantly $(\mathrm{p}<0.05)$ lower egg shape index at $10 \mathrm{ppm} \mathrm{Cu}-\mathrm{NP}$ supplemental level as compared to control while the reduced level of organic $\mathrm{Cu}$ could maintain the shape index. The findings are in agreement with Saleh et al. [45] and Yenice et al. [39] who reported no significant effect of organic minerals mixture on egg shape index.

Even at reduced supplemental levels of $\mathrm{Cu}$ from organic or nanoparticle sources, the egg albumen index, yolk index, yolk fat content, and yolk color Roche score were similar to that of control. The results corroborated with the findings of Saldanha et al. [31] who reported that dietary supplementation of organic minerals can be reduced up to $30 \%$ without compromising the egg quality parameters in semi-heavy layer hens. Attia et al. [40] also reported that no significant effect on egg yolk percentage, yolk index, albumen index, and shell thickness when 10,60 , and $120 \mathrm{ppm}$ of organic $\mathrm{Cu}$ was used in dual-purpose breeding hens' diets.

In the current experiment, the Haugh unit score was significantly $(\mathrm{p}<0.05)$ lower at $15 \mathrm{ppm} \mathrm{Cu}-\mathrm{NP}$ inclusion level. However, the values in all other treatment groups are comparable to control. The findings are in agreement with Attia et al. [40] who noticed no significant difference in Haugh unit score of egg in laying hens fed diet supplemented with 10,60, and $120 \mathrm{ppm}$ organic $\mathrm{Cu}$. Yenice et al. [39] and Stefanello et al. [28] also reported no effect of organic source of minerals on Haugh unit score in laying hens.

The eggshell thickness was significantly $(\mathrm{p}<0.05)$ highest at $15 \mathrm{ppm} \mathrm{Cu}-\mathrm{NP}$ inclusion level and also improved at 20 and $15 \mathrm{ppm}$ organic and at $10 \mathrm{ppm}$ inclusion of $\mathrm{Cu}-\mathrm{NP}$ as compared to control and other treatment groups. $\mathrm{Cu}$ plays role as cofactor of the lysyl-oxidase enzyme that is important in the formation of collagen cross-links present in the eggshell membranes [46]. Thus, the findings reinstate the improved bioavailability of both organic [13] and nano [19] forms of $\mathrm{Cu}$ over its coarse particles. Patra and Lalhriatpuii [29] reported that micro minerals nanoparticles can improve the eggshell quality in laying hens. Ramesh [33] also reported that $\mathrm{Cu}-\mathrm{NP}$ at $50 \%$ reduction level can maintain eggshell quality. Our findings are also in consistent with Stefanello et al. [28] who reported that lower egg loss, higher shell thickness, and increased strength of shell from hens fed diet supplemented with organic source of minerals as compared to inorganic source. Carvalho et al. [32] also concluded that reduction of dietary organic minerals $(\mathrm{Cu}, \mathrm{Mn}$, and $\mathrm{Zn})$ supplementation at $30 \%$ of inorganic source has comparable egg shell quality.

\section{Fertility, hatchability, and chick's quality}

The pooled data over the periods showed that the fertility rate is both dose - and form-dependent and affected particularly by $\mathrm{Cu}-\mathrm{NP}$. The fertility was significantly $(\mathrm{p}<0.05)$ higher in $\mathrm{T}_{2} 20 \mathrm{ppm}$ organic $\left.\mathrm{Cu}\right)$ group as compared to control and lower in $\mathrm{T}_{5}, 15 \mathrm{ppm}$ nano $\mathrm{Cu}$ ) as compared to $\mathrm{T}_{2}$ and $\mathrm{T}_{3}$, but comparable with all other treatment groups. Studies indicated that feeding breeder hens with organic trace minerals can improve egg quality, fertility, and hatchability [47-49], and spermatozoa quality [34] which may be due to its greater bioavailability [50]. Our findings showed that 50 and $75 \%$ (10 and $15 \mathrm{ppm}$ ) supplemental level of organic $\mathrm{Cu}$ can maintain the egg fertility rate and significantly $(\mathrm{p}<0.05)$ improves it at $100 \%$ supplemental level as compared to standard recommendation of inorganic $\mathrm{CuSO}_{4}$. Our result is consistent with the findings of Attia et al. [44] who concluded that $10 \mathrm{ppm}$ organic $\mathrm{Cu}$ is adequate for reproductive performance and egg quality in laying hens. The ICAR [23] recommended level for $\mathrm{Cu}$ is $20 \mathrm{ppm}$; therefore, the $\mathrm{Cu}$ supplementation from organic source can be reduced by $50 \%$ without affecting fertility and hatchability. The highest level of Cu-NP associated with the lowest fertility rate indicates that the $\mathrm{Cu}-\mathrm{NP}$ can reduce the egg fertility rate. Very few reports are available in literature to demonstrate the effect of $\mathrm{Cu}-\mathrm{NP}$ on egg 
fertility. However, Petruska et al. [51] and Sariozkan et al. [52] reported that nanoparticles can have spermatotoxic and/or oocyte toxic effects due to DNA fragmentation.

The hatchability rate based on total egg set was found to be better $(\mathrm{p}<0.05)$ in $\mathrm{T}_{2}$ and $\mathrm{T}_{3}$ as compared to $\mathrm{T}_{4}$ and $\mathrm{T}_{5}$ treatment groups, but all are comparable with control. The hatchability percentage based on fertilized egg set was significantly $(p<0.05)$ affected by source and dose of $\mathrm{Cu}$ which was higher in $\mathrm{T}_{3}, \mathrm{~T}_{5}$, and $\mathrm{T}_{6}$ as compared to control. Surprisingly, $\mathrm{T}_{5}$ with the lowest fertility rate $(81.70 \%)$ is having significantly $(\mathrm{p}<0.05)$ highest $(94.23 \%)$ hatchability among all treatment groups.

The significantly $(\mathrm{p}<0.05)$ lower chick mortality after hatching before-sorting in $\mathrm{T}_{5}$ and $\mathrm{T}_{6}$ dietary treatments as compared to control contributed for higher hatchability rate in $\mathrm{T}_{5}$. Similarly, the embryonic mortality rate was also significantly $(\mathrm{p}<0.05)$ lower in $\mathrm{T}_{5}$ and $\mathrm{T}_{6}$ as compared to $\mathrm{T}_{4}$ and $\mathrm{T}_{7}$. Therefore, losses of fertile egg were significantly reduced and hatchability improved at $15 \mathrm{ppm} \mathrm{Cu}-\mathrm{NP}$ inclusion level. The hatchability was improved in all nanoparticles treatment groups $\left(\mathrm{T}_{5}, \mathrm{~T}_{6}\right.$, and $\left.\mathrm{T}_{7}\right)$ but significantly $(\mathrm{p}<0.05)$ highest in $\mathrm{T}_{5}$ as compared to control. The above findings indicate that dietary supplementation of $\mathrm{Cu}-\mathrm{NP}$ can improve hatchability due to reduced embryonic mortality rate. The study revealed that $\mathrm{Cu}-\mathrm{NP}$ at $25 \%$ inclusion level of $\mathrm{CuSO}_{4}$ normal form can maintain the hatchability and increased with incremental $\mathrm{Cu}$ level. Yenice et al. [39] reported that organic minerals had no significant effect on total hatchability and fertility but can significantly $(p<0.05)$ improve hatchability of fertilized egg. In the current study, the organic $\mathrm{Cu}$ improved the fertility, hatchability, and progenies quality as well. Similar findings were reported by Saber et al. [53] who observed improved hatching and progenies performance when inorganic minerals mixture was replaced with $50 \%$ level of organic source in broiler breeder hens.

The chick quality evaluation score was better with increased supplemental dose of organic or nanoparticle of $\mathrm{Cu}$. All the groups supplemented with nanoparticles $\left(\mathrm{T}_{5}, \mathrm{~T}_{6}\right.$, and $\left.\mathrm{T}_{7}\right)$ scored significantly $(p<0.05)$ higher values as compared to control. The data showed that the chick quality can be supported at 50 and $75 \%$ reduced level of organic and nanoparticles forms of $\mathrm{Cu}$ compared to inorganic $\mathrm{CuSO}_{4}$, respectively, and can improve with higher supplemental level. The improved hatchability and chick quality are the result of novel properties of $\mathrm{Cu}-\mathrm{NP}$ such as antimicrobial effects [9] and its important role in enzyme (cytochrome oxidase) that are vital in the cellular energy generation [4]. It is hypothesized that $\mathrm{Cu}-\mathrm{NP}$ can affect the metabolic rate, thereby increasing oxygen $\left(\mathrm{O}_{2}\right)$ consumption, improve embryogenesis, hatchability, and consequently the chick growth rate [54]. It was reported that $\mathrm{Cu}-\mathrm{NP}$, especially during egg incubation period can stimulate the metabolic rate of broiler embryos to a greater level than do bulk form of $\mathrm{CuSO}_{4}$. This could be associated with that $\mathrm{Cu}-\mathrm{NP}$ has stronger bioactivity affecting the blood vessel formation and growth to a greater degree than $\mathrm{CuSO}_{4}$ [20]. The survivability rate of hens was affected in $\mathrm{T}_{3}$, but comparable with control group.

\section{Conclusion}

It was concluded that the supplemental $\mathrm{Cu}$ level $(20 \mathrm{ppm})$ in the form of inorganic $\mathrm{CuSO}_{4}$ in the diets can be reduced to 50 and $25 \%$ when $\mathrm{Cu}$ supplemented in organic $(10 \mathrm{ppm})$ and nanoparticle forms $(5 \mathrm{ppm})$, respectively, without any adverse effect on productive performance, egg quality characteristics, and hatchability of Swarnadhara breeder hens. The egg fertility, shell thickness, and body weight gain were better at $20 \mathrm{ppm} \mathrm{Cu}$ inclusion in organic form. The $\mathrm{Cu}-\mathrm{NP}$ has better effect in respect of FCR, egg weight, and shell thickness at 15 and $10 \mathrm{ppm}$ inclusion level. The egg fertility rate was not affected by treatment diets than control, while hatchability of fertilized egg and progeny quality improved. Future studies should aim to assess $\mathrm{Cu}$ retention in the body tissues or egg to ascertain the bioavailability of $\mathrm{Cu}$ from different $\mathrm{Cu}$ sources or forms.

\section{Authors' Contributions}

TMP: Conceived and designed the research. AN: Performed the experiment and nalyzed the data. TMP, $\mathrm{AN}, \mathrm{JN}, \mathrm{BNS}$, and HCI: Interpreted the data. AN, JN, BNS, and HCI: Drafted the manuscript. TMP: Edited the manuscript. All authors read and approved the final manuscript.

\section{Acknowledgments}

The first author would like to thank India-Afghan Fellowship Program, the ICAR, New Delhi for providing financial support to pursue Ph.D.

\section{Competing Interests}

The authors declare that they have no competing interests.

\section{Publisher's Note}

Veterinary World remains neutral with regard to jurisdictional claims in published institutional affiliation.

\section{References}

1. Collins, J.F., Prohaska, J.R. and Knutson, M.D. (2010) Metabolic crossroads of iron and copper. Nutr. Rev., 68(3): 133-147.

2. Hatori, Y. and Lutsenko, S. (2018) The role of copper chaperone Atox 1 in coupling redox homeostasis to intracellular copper distribution. Antioxidants, 5(3): 25-41.

3. Ognik, K., Kozłowski, K., Stępniowska, A., Szlązak, R., Tutaj, K., Zduńczyk, Z. and Jankowski, J. (2019) The effect of manganese nanoparticles on performance, redox reactions and epigenetic changes in turkey tissues. Animals, 13(6): 1137-1144.

4. Pineda, L., Sawosz, E., Vadalasetty, K.P. and Chwalibog, A. (2013) Effect of copper nanoparticles on metabolic rate and 
development of chicken embryos. Anim. Feed Sci. Technol., 186(1-2): 125-129.

5. Wu, X.Z., Zhang, T.T., Guo, J.G., Liu, Z., Yang, F.H. and Gao, X.H. (2015) Copper bioavailability, blood parameters and nutrient balance in mink1. J. Anim. Sci., 93(1): 176-184.

6. Zhao, J., Shirley, R., Vazquez-Anon, J.J., Dibner, J.D., Richards, P., Fisher, T., Al-Lard, J.P. and Giesen, A.F. (2010) Effects of chelated trace minerals on growth performance, breast meat yield, and foot pad health in commercial meat broilers. J. Appl. Poult. Res., 19(4): 365-372.

7. Pakel, A.Y. and Alp, M. (2011) Effects of different dietary copper sources on laying hen performance and egg yolk cholesterol. J. Appl. Poult. Res., 20(4): 506-513.

8. Nys, Y., Révy, P.S. and Jondreville, C. (2003) Zinc, cuivre et manganèse en aviculture: Rôle, disponibilité et risque pour l'environnement. In: Cinquièmes Journées de la Recherche Avicole, Tours. p1-12.

9. Scott, A., Prasad, K., André, V.C. and Sawosz, E. (2018) Copper nanoparticles as an alternative feed additive in poultry diet: A review. Nanotech. Rev., 7(1): 69-93.

10. Paik, I.K., Seo, S.H., Um, J.S., Chang, M.B. and Lee, B.H. (1999) Effects of Supplementary copper-chelate on the performance and cholesterol level in plasma and breast muscle of broiler chickens. Asian-Australas. J. Anim. Sci., 12(5): 794-798.

11. Dozier, W.A., Davisa, J., Freeman, M.E. and Ward, T.L. (2003) Early growth and environmental implications of dietary zinc and copper concentrations and sources of broiler chicks. Br. Poult. Sci. J., 44(5): 726-731.

12. Ferket, P., van Heugten, E. and Angel, R. (2002) Nutritional strategies to reduce environmental emissions from non-ruminants. J. Anim. Sci., 80(2): 168-182.

13. Raje, K., Ojha, S., Mishra, A., Munde, V.K., Rawat, C. and Chaudhary, S.K. (2018) Impact of supplementation of mineral nano-particles on growth performance and health status of animals: A review. J. Entomol. Zool. Stud., 6(3): 1690-1694.

14. Coppenet, M., Golven, J., Simon, J.C., Le Corre, L. and Le Roy, M. (1993) Chemical evolution of soils in intensive animal-rearing farms: the example of Finnish. Agronomie, 13(2): 77-83.

15. Nollet, L., van der Klis, J.D., Lensing, M. and Spring, P. (2007) The effect of replacing inorganic with organic trace minerals in broiler diets on productive performance and mineral excretion. J. Appl. Poult. Res., 16(4): 592-597.

16. Hefnawy, A.E. and El-Khaiat, H. (2015) Copper and animal health (importance, maternal fetal, immunity and DNA relationship, deficiency and toxicity). Int. J. Agro. Vet. Med. Sci., 9(5): 195-211.

17. Gheisari, A.A., Sanei, A., Samie, A., Gheisari, M.M. and Toghyani, M. (2011) Effect of diets supplemented with different levels of manganese, zinc, and copper from their organic or inorganic sources on egg production and quality characteristics in laying hens. Biol. Trace Elem. Res., 142(3): 557-571.

18. Wang, Z.L. (2000) Characterizing the structure and properties of individual wire-like nanoentities. Adv. Mater., 12(17): 1295-1298.

19. Tamilvanan, A., Balamurugan, K., Ponappa, K. and Kumar, B.M. (2014) Copper nanoparticles: Synthetic strategies, properties and multifunctional application. Int. J. Nanosci., 13(2): 143.

20. Mroczek-Sosnowska, N., Sawosz, E., Vadalasetty, K., Łukasiewicz, M., Niemiec, J., Wierzbicki, M., Kutwin, M., Jaworski, S. and Chwalibog, A. (2015) Nanoparticles of copper stimulate angiogenesis at systemic and molecular level. Int. J. Mol. Sci., 16(3): 4838-4849.

21. Al-Bairuty, G.A., Boyle, D., Henry, T.B. and Handy, R.D. (2016) Effects of copper sulphate compared to copper nanoparticles in rainbow trout (Oncorhynchus mykiss) at low pH: Physiology and metal accumulation. Aquat. Toxicol., 177: 188-198.
22. Kozłowski, K., Jankowski, J., Otowski, K., Zduńczyk, Z. and Ognik, K. (2018) Metabolic Parameters in young turkeys fed diets with different inclusion levels of copper nanoparticles. Pol. J. Vet. Sci., 21(2): 245-253.

23. ICAR. (2013) Nutrients Requirement of Animal-Poultry. Indian Council of Agriculture Research, New Delhi.

24. Haugh, R.R. (1937) The Haugh unit for measuring egg quality. U. S. Egg Poult. Mag., 43: 552-573.

25. Roche Company. (1996) Roce Yolk Colour Fan No. 1155 Printed in Switzerland, DF, Bornstein and Bartov. Roche Company, Chennai.

26. AOAC. (2012) Official Methods of Analysis. 18 ${ }^{\text {th }}$ ed. Association of Official Analytical Chemists, Washington, DC.

27. Tona, K., Bamelis, F., De-Ketelaere, B., Bruggeman, V., Moraes, V.M.B., Buyse, J., Onagbesan, O. and Decuypere, E. (2003) Effects of egg storage time on spread of hatch, chick quality and chick juvenile growth. Poult. Sci., 82(5): 736-741.

28. Stefanello, C., Santos, T. C., Murakami, A. E., Martins, E.N. and Carneiro, T.C. (2014) Productive performance, eggshell quality, and eggshell ultrastructure of laying hens fed diets supplemented with organic trace minerals. Poult. Sci., 93(1): 104-113.

29. Patra, A. and Lalhriatpuii, M. (2019) Progress and prospect of essential mineral nanoparticles in poultry nutrition and feeding-a review. Biol. Trace Elem. Res., 197(1): 233-253.

30. NRC. (1994) Nutrient Requirements of Poultry. $9^{\text {th }}$ ed. National Academic Press, Washington, DC.

31. Saldanha, E.S.P., Garcia, E.A., Pizzolante, C.C., Faittarone, A.B.G., da Sechinato, A., Molino, A.B. and Laganá, C. (2009) Effect of organic mineral supplementation on the egg quality of semi-heavy layers in their second cycle of lay. Braz. J. Poult. Sci., 12(4): 241-247.

32. Carvalho, L.S.S., Rosa, D.R.V., Litz, F.H., Fagundes, N.S. and Fernandes, E.A. (2015) Effect of the inclusion of organic copper, manganese, and zinc in the diet of layers on mineral excretion, egg production, and eggshell quality. Braz. J. Poult. Sci., 17(9): 87-92.

33. Ramesh, J. (2014) Effect of Nanomineral Supplementation in TANUVAS Smart Mineral Mixture on the Performance of Lambs (Doctoral Dissertation, Tamil Nadu Veterinary and Animal Sciences University), Chennai, Tamil Nadu, India.

34. Londero, A., Rosa, A.P., Luiggi, F.G., Fernandes, M.O., Guterres, A., de Moura, S., Pedroso, N.H. and Santos, N. (2020) Effect of supplementation with organic and inorganic minerals on the performance, egg and sperm quality and, hatching characteristics of laying breeder hens. Anim. Reprod. Sci., 215: 106309.

35. Hill, E.G., Johnson, S.B., Lawson, L.D., Mahfouz, M.M. and Holman, R.T. (2000) Perturbation of the metabolism of essential fatty acids by dietary partially hydrogenated vegetable oil. Proc. Natl. Acad. Sci. U. S. A., 79(4): 953-957.

36. Shankar, S. and Rhim, J.W. (2014) Effect of copper salts and reducing agents on characteristics and antimicrobial activity of copper nanoparticles. Mater. Lett., 132: 307-311.

37. Duran, N., Marcato, P.D., De-Conti, R., Alves, O.L., Costa, F.T.M. and Brocchi, M. (2010) Potential use of silver nanoparticles on pathogenic bacteria, their toxicity, and possible mechanisms of action. J. Braz. Chem. Soc., 21(16): 949-959.

38. Prabhu, B.M., Ali, S.F., Murdock, R.C., Hussain, S.M. and Srivatsan, M. (2010) Copper nanoparticles exert size and concentration dependent toxicity on somatosensory neurons of rat. Nanotoxicology, 4(2): 150-160.

39. Yenice, E., Mizrak, C., Gültekin, M., Zafer, A.T.İ.K. and Tunca, M. (2015) Effects of dietary organic or inorganic manganese, zinc, copper and chrome supplementation on the performance, egg quality and hatching characteristics of laying breeder hens. Ankara Üniv. Vet. Fakültesi Derg., 62(1): 63-68.

40. Attia, Y.A., Abdalah, A.A., Zeweil, H.S., Bovera, F., 
El-Din, A.T. and Araft, M.A. (2011) Effect of inorganic or organic copper additions on reproductive performance, lipid metabolism and morphology of organs of dual-purpose breeding hens. Arch. Geflugelkunde, 75(3): 169-178.

41. Lim, H.S. and Paik, I.K. (2006) Effects of dietary supplementation of copper chelates in the form of methionine, chitosan and yeast in laying hens. Asian-Australas. J. Anim. Sci., 19(8): 1174-1178.

42. Pesti, G.M. and Bakalli, R.I. (1998) Studies on the feeding cupric sulfate pentahydrate to laying hens on egg cholesterol content. Poult. Sci., 77(10): 1540-1545.

43. Sawosz, E., Łukasiewicz, M., Łozicki, A., Sosnowska, M., Jaworski, S., Niemiec, J. and Chwalibog, A. (2018) Effect of copper nanoparticles on the mineral content of tissues and droppings, and growth of chickens. Arch. Anim. Nutr., 72(5): 396-406.

44. Attia, Y.A., Qota, E.M., Zeweil, H.S., Bovera, F., Abd Al-Hamid, A.E. and Sahledom, M.D. (2012) Effect of different dietary concentrations of inorganic and organic copper on growth performance and lipid metabolism of White Pekin male ducks. Br. Poult. Sci., 53(1): 77-88.

45. Saleh, A.A., Eltantawy, M.S., Gawish, E.M., Younis, H.H., Amber, K.A., Abd El-Moneim, A.E.M. and Ebeid, T.A. (2019) Impact of dietary organic mineral supplementation on reproductive performance, egg quality characteristics, lipid oxidation, ovarian follicular development, and immune response in laying hens under high ambient temperature. Biol. Trace Elem. Res., 195(2): 506-514.

46. Chowdhury, S.D. (1990) Shell membrane system in relation to lathyrogen toxicity and copper deficiency. World Poult. Sci. J., 46(2): 153-169.

47. Mabe, I., Rapp, C., and Bain, M.M. and Nys, Y. (2003) Supplementation of a corn-soybean meal diet with manganese, copper, and zinc from organic or inorganic sources improves eggshell quality in aged laying hens. Poult. Sci., 82(12): 1903-1913.

48. Tang, P., Patterson, P.H. and Puri, V.M. (2006) Effect of feed segregation on the commercial hen and egg quality. J. Appl. Poult. Res., 15(4): 564-573.

49. Uni, Z., Yadgary, L. and Yair, R. (2012) Nutritional limitations during poultry embryonic development. J. Appl. Poult. Res., 21(1): 175-184.

50. Nollet, L., Huyghebaert, G. and Spring, P. (2008) Effect of different levels of dietary organic (Bioplex) trace minerals on live performance of broiler chickens by growth phases. J. Appl. Poult. Res., 17(1): 109-115.

51. Petruska, P., Capcarova, M. and Sutovsky, P. (2014) Antioxidant supplementation and purification of semen for improved artificial insemination in livestock species. Turk. J. Vet. Anim. Sci., 38(6): 643-652.

52. Sariozkan, S., Bucak, M.N., Tuncer, P.B., Buyukleblebici, S., Eken, A. and Akay, C. (2015) Influence of fetuin and hyaluronan on the post-thaw quality and fertilizing ability of Holstein bull semen. Cryobiology, 71(1): 119-124.

53. Saber, S., Kutlu, H.R., Yusuf, U.Z., Celik, L., Yucelt, O. and Baylan, M. (2020) Effects of form of dietary trace mineral premix on fertility and hatchability of broiler breeder hens and post-hatch performance and carcass parameters of their progenies. Kafkas Üniv. Vet. Fakültesi Derg., 26(2): 171-180.

54. Scott, A., Vadalasetty, K.P., Sawosz, E., Łukasiewicz, M., Vadalasetty, R.K.P., Jaworski, S. and Chwalibog, A. (2016) Effect of copper nanoparticles and copper sulphate on metabolic rate and development of broiler embryos. Anim. Feed Sci. Technol., 220: 151-158.

$* * * * * * * *$ 